\title{
High-pressure phases of sulfur: crystal structure prediction and topological analysis
}

Pavel N.Gavryushkin ${ }^{\text {a,b, }}$, Zakhar I. Popov ${ }^{c}$, Litasov D. Konstantin ${ }^{\text {a,b }}$, Sergey S. Dobrosmislov

$$
\text { d, Gavryushkin Alex }{ }^{\text {e }}
$$

a V. S. Sobolev Institute of Geology and Mineralogy, Russian Academy of Science, Siberian

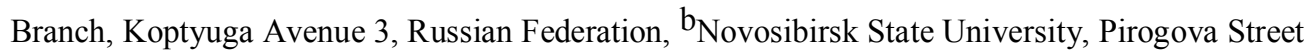
2, Novosibirsk, 630090, Russian Federation, ${ }^{\mathrm{c}}$ National University of Science and Technology MISIS 4 Leninskiy pr., 119049 Moscow, Russian Federation, and ${ }^{\mathrm{d}}$ Siberian Federal University, 79 Svobodny pr., Krasnoyarsk, 660041, Russian Federation, e Department of Computer Science, University of Auckland, New Zealand.

*Correspondence e-mail: p.gavryushkin@gmail.com.

\begin{abstract}
High-pressure behaviour of sulfur above $100 \mathrm{GPa}$ is studied with density functional theory (DFT) and evolutionary structure prediction algorithms. Based on DFT calculations energetical relations between experimentally fixed S-III, S-V and $\beta$-Po are determined. S-V is found to be the lower pressure phase transforming to S-III at $90 \mathrm{Gpa}$. The second order character of transformation from S-III to $\beta$-Po is theoretically approved and calculations yield a value of around $300 \mathrm{Gpa}$ for the transition pressure at $0 \mathrm{~K}$. $\beta$-Po structure is not stable in trigonal symmetry below this pressure and spontaneously transforms in monoclinic structure identical to S-III. The calculations yield values of $333 \mathrm{Gpa}$ and $460 \mathrm{Gpa}$ for the transitions from $\beta$-Po to simple cubic and from simple cubic to body centered cubic structures respectively. Crystal structure prediction method let to reveal a new phase, named S-V(II), which is energetically more favourable than S-III above $100 \mathrm{GPa}$.
\end{abstract}

\section{Introduction}

High pressure investigation of sulfur as well as other group VI elements attracts considerable interest of physicists and crystallographers due to metallization of the initially covalent S-S bonds ${ }^{1-3}$, transition from insulator through semiconductor to superconductor ${ }^{4-6}$, occurrence of several monoclinic and triclinic phases ${ }^{7,8}$, pressure dependent incommensurate modulations ${ }^{7,8}$ and amorphization 2, 3,9,10. Being the leader among elements by amount of different polymorphs under ambient conditions ${ }^{11}$ it is probably preserves among leaders in high pressure region, although the number of polymorphs is drastically decreases. 
The well-known orthorhombic structure of $\alpha-\mathrm{S}$, comprising of $\mathrm{S}_{8}$ rings, ${ }^{12}$ is stable up to 20-30 Gpa and became gradually amorphous on further compression ${ }^{2,3,10}$. Recrystallization starts around $37 \mathrm{Gpa}^{3}$ and fully completes only at $75 \mathrm{Gpa}^{3}$. Sulfur recrystallize in structure with tetragonal helic $\left(4_{1}\right.$ and $\left.4_{3}\right)$ chains of sulfur atoms ${ }^{13,14}$, named S-II. Unfortunately, different authors use different numerical notations of high-pressure phases of sulfur. For example, the same phase denoted as S-III by Hejny and co-authors ${ }^{7}$ and as S-IV by Degtyareva and coauthors ${ }^{8}$. In this work we will follow the notation of Hejny and co-authors ${ }^{7}$. Further compression of S-II leads to crystallizations of S-III at 83-86 GPa. Based on assumption on orthorhombic symmetry and type of centering of the unit cell this phase was named bco (bodycentered orthorhombic). Zakharov and co-authors ${ }^{4}$ were first who indicate on probable mistake in symmetry determination of S-III, showed that in the orthorhombic model the energy of this phase is higher than the energy $\beta$-Po in all pressure range. Then Nishikawa and coauthors ${ }^{15}$ based on symmetry and density of state considerations make assumption about monoclinic character of S-III. This assumption was experimentally confirmed by Hejny and co-authors ${ }^{7}$ and Degtyareva and co-authors ${ }^{14}$. In addition the incommensurately modulated character of S-III was revealed in these experiments. As the assumption about orthorhombic character of S-III turns out to be wrong, bco designation was changed to $\mathrm{bcm}$ (body-centered monoclinic). Our results on crystal structure prediction confirms a monoclinic character of this phase. The additional triclinic phase S-V, appearing on decompression of S-III around $90 \mathrm{GPa}$ and traced down to $62 \mathrm{Gpa}$, was fixed by Hejny and co-authors ${ }^{7}$. Structural relations of S-V phase with SIII was not still analysed. We present this analysis complemented by ab-initio calculations in the present work. On further compression S-III transforms to $\beta$-Po at $153-162 \mathrm{Gpa}^{3,8}$. $\beta$-Po was experimentally traced up to $212 \mathrm{Gpa}^{8}$ and further high-pressure region is investigated only theoretically. According to theoretical results $\beta$-Po structure first transforms to simple cubic (sc) structure and then to body-centered cubic $(b c c)$ structure at 280 Gpa and 540 Gpa respectively 5 , 6.

\section{Details of calculations}

Crystal structure prediction performed on the basis of evolutionary algorithms implemented in USPEX (Universal Structure Predictor: Evolutionary Xtallography) package ${ }^{16-}$ ${ }^{20}$. Local optimization performed in the frame of DFT theory with VASP code ${ }^{21,22}$, using the plane wave basis set and projector augmented wave method ${ }^{23}$. Exchange-correlation effects were taken into account in the generalized gradient approximation (GGA) by the PerdewBurke-Ernzerhof (PBE) functional ${ }^{24}$. For the crystal structure searches, we used a plane-wave basis set cutoff of $350 \square \mathrm{eV}$ and performed the BZ integrations using uniform Gamma-centred k- 
point meshes with a k-point grid of spacing $2 \pi \times|0.035| \square \AA^{-1}$. Iterative relaxation of atomic positions was stopped when all forces were smaller than $0.001 \square \mathrm{eV} \square \AA^{-1}$. Calculations were carried out for 100, 200, 300, 400 and $500 \mathrm{GPa}$ with 8 atoms per unit cell. Temperature in all calculations was $0 \mathrm{~K}$. Phonon dispersion curves were calculated in PHONOPY package ${ }^{25}$. Topological analysis was performed with TOPOSPro package ${ }^{26-28}$.

\section{Results and discussion}

\section{Structures descriptions and ab-initio calculations of S-III, S-V and $\beta$-Po structures}

In S-III, S-V and $\beta$-Po structures each sulfur atom has six strong and eight weak contacts with neighbouring atoms. The structure representation corresponding to the first set of bonds is the stacking of ideal, in the case of $\beta$-Po, or deformed, in the cases of S-III and S-V rhombohedrons. Structure representation corresponding to the second set of bonds is the stacking of deformed body-centered cubes. The second structure representation is useful for crystallographic description of the transitions from this family of structures to $b c c$ structure, as in such representation this transitions corresponds to transformation of deformed $b c c$ structure to ideal one. The first representation is useful for description of transitions between closely related S-III, S-V and $\beta$-Po structures.

To transform rhombohedral $\beta$-Po structure into S-III, it should be stretched along one of the two-fold axes or mirror planes. As the result of such deformation the symmetry decreases from trigonal to monoclinic and the lengths of the edges adopt two different values: $2.208 \AA$ and $2.225 \AA$ (Fig. 1a). The difference between length of edges of such pseudorhombohedron does not exceed $1 \%$ of the smallest value. As well as S-III, S-V can be represented as deformed $\beta$-Po structure, but the deformation is more complex in this case and reduce trigonal symmetry to triclinic. The difference between pseudorhombohedron edges of $\mathrm{S}-\mathrm{V}$ is much higher than that for S-III and achieves $18 \%$ (Fig. $1 \mathrm{~b}$ ).

Figure 1. Deformed rhombohedrons outlined by the strong bonds of structures (a) S-III, (b) S-V, (c) S-V(II). Numbers under bonds corresponds to bond lengths in $\AA$. Structural data are taken from ${ }^{29}$ for S-III and from our calculations for S-V(II).

To clarify the influence of deformation on energy of $\beta$-Po structures, we perform ab-initio density functional theory calculations on $\mathrm{S}-\mathrm{III}^{7}, \mathrm{~S}-\mathrm{V}^{7}$ and $\beta-\mathrm{Po}^{8}$ structures in the pressure range 0-500 GPa. The effect of modulations was not concerned and calculations of S-III structure were 
performed with average model. The analysis of phases is limited to the lower boundary on pressure around 30-40 Gpa. Below this pressure structures became unstable and their energy starts to increase rapidly. This can be clearly seen from dependence of cell parameters of S-III on pressure which is presented in Supplementary. This probably corresponds to amorphization, which was experimentally fixed nearly at the same pressure. The transition from S-III to S-V was reproduced at $90 \mathrm{Gpa}$, but the energy difference between S-III and S-V is very small, around several thousandths of $\mathrm{eV}$. This small difference explain the joint occurrence of S-V and S-III in high-pressure experiments. As was shown by Nishikawa ${ }^{1}$ the energy of modulated S-III structure is lower than that of unmodulated S-III, hence the transformation from S-V to modulated S-III should be slightly lower than presented value, $90 \mathrm{GPa}$. Taking into account this correction the calculated value of pressure for transition from S-V to S-III is in good agreement with experimental value, 83-86 Gpa.

Optimization of $\beta$-Po structure shows that in trigonal symmetry it can exist only above 300 Gpa, below this pressure the symmetry of the optimized structure is monoclinic with space group $C 2 / m$ (Fig.2). This monoclinic structure is identical to S-III. For comparison, at 100 Gpa the space group and unit cell parameters of monoclinic $\beta$-Po is $C 2 / m, a=2.769 \AA, b=3.445 \AA$, $\mathrm{c}=2.261 \AA, \beta=112.483^{\circ}$ and of S-III is $C 2 / \mathrm{m}, \mathrm{a}=2.804 \AA, \mathrm{b}=3.455 \AA, \mathrm{c}=2.208 \AA, \beta=113.24^{\circ}$. The deviation from trigonal symetry increases with decreasing pressure. For example, at 290 Gpa the difference between edges of rhombohedron is $0.005 \%$, at $100 \mathrm{Gpa}$ this value reaches $2.3 \%{ }^{1}$. Thus according to our calculations transition from S-III to $\beta$-Po is a continuous second-order transition, completing around $300 \mathrm{GPa}$. The second order character of this transition is confirmed by experimental results ${ }^{8}$. However, the experimental value of pressure for this transition is almost two time lower than that determined in calculations. This difference can be attributed to the influence of temperature, the effect of which was not considered in our calculations. As structural difference between S-III and $\beta$-Po is very small and slight deformation can transform one structure into another, the small contribution of entropic member into Gibbs energy can be crucial and change phase relations determined at $0 \mathrm{~K}$, made more symmetrical $\beta$-Po phase more favourable. As the result the transition pressure can be sufficiently decreased.

Figure 2. The dependence of $a$ - and $c$-axis of $\beta$-Po on pressure in rhombohedral setting; $b$-axis is equal to $c$-axis.

\footnotetext{
${ }^{1}$ This value is different from value of $1 \%$ mentioned above because this value is based on theoretical results and $1 \%$ - on experimental results
} 


\section{Crystal structure prediction}

Among three structures experimentally fixed around $100 \mathrm{Gpa}, \mathrm{S}-\mathrm{III}, \mathrm{S}-\mathrm{V}$ and $\beta$-Po, two lasts were found in our crystal structure prediction calculations at $100 \mathrm{GPa}$. In addition, one new structure, designated as $\mathrm{S}-\mathrm{V}(\mathrm{II})$, was found at this pressure. The choice of the name is explained by the fact, that this structure is characterized by the same triclinic symmetry as S-V and can be presented as deformed $\beta$-Po structure. The deformation is higher than that of S-III but less than that of S-V, thus structurally S-V(II) occupies the intermediate place between S-III and S-V (Fig.1). For example at $100 \mathrm{Gpa}$, the difference between edges of rhombohedron formed by S-S bonds is $18 \%$ for $\mathrm{S}-\mathrm{V}, 8.6 \%$ for $\mathrm{S}-\mathrm{V}$ (II), and $2.3 \%$ for S-III (Fig. 1). The new phase is more favourable than both S-V and S-III above $100 \mathrm{Gpa}$ (Fig.3). The maximum difference in energy between S-V(II) and S-III, S-V is reached around $200 \mathrm{Gpa}$ and it is equal to $0.007 \mathrm{eV} /$ atom for difference between S-V(II) and S-III, and $0.01 \mathrm{eV} /$ atom for difference between S-V(II) and S-V (Fig.3). The calculation of phonon dispersion curves, presented in the Supplementary, shows the dynamical stability of the new phase. Despite the fact, that predicted S-V(II) phase was not found in high-pressure experiments, there is a possibility of its missing in experiments due to its similarity to S-III and S-V structures and complex character of diffraction pattern of incommensurately modulated S-III. But irrespectively of possibility of experimental synthesis of $S-V(I I)$, the fact of its theoretical existence shows that two known type of deformation of $\beta$-Po structure, S-III and S-V, are not unique and exist at least one other type of deformation which is even more favourable energetically than known S-III and S-V.

Figure 3. Energetical relations of predicted and experimental phases (a) below and (b) above $300 \mathrm{Gpa}$.

The calculations at 200, 300, 400 and 500 Gpa reveal known $\beta$-Po, sc and $b c c$ as the most energetically favourable. Any new phases was not found in this calculations. The absence of the new phases in calculations at 200-500 Gpa shows that phase diagram inferred from the analogy with other group VI elements is quite complete and future synthesis of the new phases with less than 8 atoms in the unit cell in this pressure range seems unlikely. According to our calculations, that transition pressure from $\beta$-Po to $s c$ is $333 \mathrm{Gpa}$, from sc to bcc $-460 \mathrm{Gpa}$ (fig.3). While transition from $\beta$-Po to open $s c$ structure at such high pressure ${ }^{6}$ is unusual it has a strict analogy with other group VI element, polonium, which under heating to $348 \mathrm{~K}$ transforms from sc to $\beta$ - 
Po structure ${ }^{30-32}$. Thus unique for element under ambient conditions $s c$ structure has one more unique occurrence under high-pressure.

\section{Sources of funding}

We thank the Information Technology Centre of Novosibirsk State University for providing access to the cluster computational resources. The research was supported by the Russian Foundation for Basic Research through grants (No 13-0312158) and the Ministry of Education and Science of Russian Federation (No 14.B25.31.0032). The work of ZIP is supported by the Ministry of Education and Science of the Russian Federation in the framework of Increase Competitiveness Program of NUST «MISiS» (No K2-2015-033).

\section{References}

(1) Nishikawa, A. In Electronic structure of sulfur under high pressure, Journal of Physics: Conference Series, 2008; IOP Publishing: 2008; p 012008.

(2) Akahama, Y.; Kobayashi, M.; Kawamura, H., Pressure-unduced structural phase transition in sulfur at $83 \mathrm{GPa}$. Phys Rev B 1993, 48, (10), 6862-6864.

(3) Luo, H.; Greene, R. G.; Ruoff, A. L., Beta-Po phase of sulfur at 162 GPa: X-Raydiffraction study to 212 GPa. Physical Review Letters 1993, 71, (18), 2943-2946.

(4) Zakharov, O.; Cohen, M. L., Theory of structural, electronic, vibrational, and superconducting properties of high-pressure phases of sulfur. Phys Rev B 1995, 52, (17), 12572.

(5) Rudin, S. P.; Liu, A. Y.; Freericks, J.; Quandt, A., Comparison of structural transformations and superconductivity in compressed sulfur and selenium. Phys Rev B 2001, 63, (22), 224107.

(6) Rudin, S. P.; Liu, A. Y., Predicted simple-cubic phase and superconducting properties for compressed sulfur. Physical review letters 1999, 83, (15), 3049.

(7) Hejny, C.; Lundegaard, L.; Falconi, S.; McMahon, M.; Hanfland, M., Incommensurate sulfur above $100 \mathrm{GPa}$. Phys Rev B 2005, 71, (2), 020101.

(8) Degtyareva, O.; Gregoryanz, E.; Somayazulu, M.; Mao, H.-k.; Hemley, R. J., Crystal structure of the superconducting phases of S and Se. Phys Rev B 2005, 71, (21), 214104.

(9) Plasienka, D.; Martonak, R., Study of pressure-induced amorphization in sulfur using ab initio molecular dynamics. Phys Rev B 2012, 85, (9).

(10) Luo, H.; Ruoff, A. L., X-ray-diffraction study of sulfur to $32 \mathrm{GPa}$ : Amorphization at 25 GPa. Phys Rev B 1993, 48, (1), 569.

(11) Steudel, R.; Eckert, B., Solid sulfur allotropes. In Elemental Sulfur and Sulfur-Rich Compounds I, Steudel, R., Ed. 2003; Vol. 230, pp 1-79.

(12) Rettig, S.; Trotter, J., Refinement of the structure of orthorhombic sulfur, $\alpha$-S8. Acta Crystallographica Section C: Crystal Structure Communications 1987, 43, (12), 2260-2262.

(13) Fujihisa, H.; Akahama, Y.; Kawamura, H.; Yamawaki, H.; Sakashita, M.; Yamada, T.; Honda, K.; Le Bihan, T., Spiral chain structure of high pressure selenium- II' and sulfur- II from powder x-ray diffraction. Phys Rev B 2004, 70, (13), 134106.

(14) Degtyareva, O.; Gregoryanz, E.; Somayazulu, M.; Dera, P.; Mao, H.-k.; Hemley, R. J., Novel chain structures in group VI elements. Nature materials 2005, 4, (2), 152-155.

(15) Nishikawa, A.; Niizeki, K.; Shindo, K., The Structural and Electronic Properties of Sulfur under High Pressure. physica status solidi (b) 1999, 211, (1), 373-378.

(16) Glass, C. W.; Oganov, A. R.; Hansen, N., USPEX - Evolutionary crystal structure prediction. Computer Physics Communications 2006, 175, (11-12), 713-720. 
(17) Lyakhov, A. O.; Oganov, A. R.; Stokes, H. T.; Zhu, Q., New developments in evolutionary structure prediction algorithm USPEX. Computer Physics Communications 2013, $184,(4), 1172-1182$.

(18) Lyakhov, A. O.; Oganov, A. R.; Valle, M., How to predict very large and complex crystal structures. Computer Physics Communications 2010, 181, (9), 1623-1632.

(19) Oganov, A. R.; Glass, C. W., Crystal structure prediction using ab initio evolutionary techniques: Principles and applications. J. Chem. Phys. 2006, 124, (24).

(20) Oganov, A. R.; Glass, C. W.; Ono, S., High-pressure phases of CaCO3: Crystal structure prediction and experiment. Earth Planet Sc Lett 2006, 241, (1-2), 95-103.

(21) Kresse, G.; Furthmuller, J., Efficiency of ab-initio total energy calculations for metals and semiconductors using a plane-wave basis set. Comput. Mater. Sci. 1996, 6, (1), 15-50.

(22) Kresse, G.; Joubert, D., From ultrasoft pseudopotentials to the projector augmented-wave method. Phys Rev B 1999, 59, (3), 1758-1775.

(23) Blöchl, P. E., Projector augmented-wave method. Phys Rev B 1994, 50, (24), 17953.

(24) Perdew, J. P.; Burke, K.; Ernzerhof, M., Generalized gradient approximation made simple. Physical review letters 1996, 77, (18), 3865.

(25) Togo, A.; Oba, F.; Tanaka, I., First-principles calculations of the ferroelastic transition between rutile-type and $\mathrm{CaCl}$ 2-type $\mathrm{SiO} 2$ at high pressures. Phys Rev B 2008, 78, (13), 134106. (26) Blatov, V. A.; Shevchenko, A. P.; Proserpio, D. M., Applied topological analysis of crystal structures with the Program Package ToposPro. Cryst Growth Des 2014, 14, (7), 35763586.

(27) Blatov, V. A., Multipurpose crystallochemical analysis with the program package TOPOS. IUCr CompComm Newsletter 2006, 7, (4).

(28) Blatov, V. A., Nanocluster analysis of intermetallic structures with the program package TOPOS. Structural Chemistry 2012, 23, (4), 955-963.

(29) Hejny, C.; McMahon, M., Complex crystal structures of Te-II and Se-III at high pressure. Phys Rev B 2004, 70, (18), 184109.

(30) Beamer, W. H.; Maxwell, C. R., Physical Properties of Polonium. II. X-Ray Studies and Crystal Structure. The Journal of Chemical Physics 1949, 17, (12), 1293-1298.

(31) DeSando, R.; Lange, R., The structures of polonium and its compounds $-\mathrm{I} \alpha$ and $\beta$ polonium metal. Journal of Inorganic and Nuclear Chemistry 1966, 28, (9), 1837-1846.

(32) Maxwell, C. R., Physical Properties of Polonium. I. Melting Point, Electrical Resistance, Density, and Allotropy. The Journal of Chemical Physics 1949, 17, (12), 1288-1292. 